\title{
AVALIAÇÃO DO POTENCIAL DE REGENERAÇÃO DE RAÍZES DE MUDAS DE Pinus taeda L., PRODUZIDAS EM DIFERENTES TIPOS DE RECIPIENTES, E O SEU DESEMPENHO NO CAMPO ${ }^{1}$
}

\author{
Adalberto Brito de Novaes², José Geraldo de Araújo Carneiro³, Deborah Guerra Barroso ${ }^{4}$ e \\ Paulo Sérgio dos Santos Leles ${ }^{5}$
}

\begin{abstract}
RESUMO - Avaliou-se a qualidade de mudas de Pinus taeda com base no estudo do potencial de regeneração de raízes (P.R.R.), visando alcançar altas taxas de sobrevivência e crescimento inicial após o plantio. No viveiro, foram empregados cinco tratamentos: a) blocos prensados com $10 \mathrm{~cm}$; b) blocos prensados com $7 \mathrm{~cm}$; c) raiz nua; d) tubetes D48 (menor densidade); e) tubetes D96 (maior densidade). No laboratório avaliou-se o P.R.R. em aquários e caixas, por meio da determinação dos seguintes parâmetros: a) número total de raízes regeneradas em aquários; b) número total de raízes regeneradas maiores que $1 \mathrm{~cm}$, em aquários; e c) comprimento total de raízes regeneradas em aquários e caixas (cm). No campo, foram avaliados a sobrevivência e o crescimento inicial através das medições de altura da parte aérea e do diâmetro do caule ao nível do solo. O P.R.R. foi considerado um parâmetro fisiológico confiável na determinação da qualidade de mudas de Pinus taeda e na previsão de seu desempenho no campo. O comprimento total e o número de raízes novas maiores que $1 \mathrm{~cm}$ apresentaram correlações significativas com o desempenho das mudas no campo. As mudas de Pinus taeda de melhor padrão de qualidade, com altos valores de P.R.R. e melhor desempenho no campo 24 meses após o plantio, foram produzidas em blocos prensados com $10 \mathrm{~cm}$. As médias mais baixas, para os parâmetros avaliados em laboratório e no campo, foram verificadas em mudas produzidas em tubetes.
\end{abstract}

Palavras-chave: $\quad$ Pinus taeda, potencial de regeneração de raízes (P.R.R.) e mudas.

\section{EVALUATION OF THE ROOT GROWTH POTENTIAL OF Pinus taeda L. SEEDLINGS PRODUCED IN DIFFERENT TYPES OF RECIPIENTS, AND THEIR PERFORMANCE IN THE FIELD}

\begin{abstract}
The quality of Pinus taeda seedlings, produced in different types of containers, was evaluated based on root regeneration potential (RRP), to obtain seedling production with high survival rates and initial growth after planting. The study was conducted under greenhouse conditions, using five treatments: a) pressed blocks with $10 \mathrm{~cm}$; b) $7 \mathrm{~cm}$ pressed blocks; c) bare root; d) D48 tubes (lower density); and e) D96 tubes (higher density). RRP in aquariums and boxes, was evaluated using the following parameters: a) total number of roots regenerated in aquariums; $b$ ) total number of regenerated roots $>1 \mathrm{~cm}$ in aquariums; $c$ ) total length of roots regenerated in aquariums and boxes (cm). Seedling survival and initial growth were evaluated by measuring height of the aerial part and stem diameter at ground level. RRP was found to be a reliable physiological parameter to determine the quality of Pinus taeda seedling and to predict its performance in the field. Total length and number of new roots $>1 \mathrm{~cm}$ showed significant correlations with the performance of the seedlings in the field. The Pinus taeda seedlings showing better quality for all the studied parameters, and field performance 24 months after planting were produced in $10 \mathrm{~cm}$ pressed blocks. The lowest averages for all parameters evaluated were observed in seedlings produced in tubes.
\end{abstract}

Key words: $\quad$ Pinus taeda, root regeneration potential (RRP), seedlings.

1 Recebido para publicação em 28.10.2000.

Aceito para publicação em 3.12.2002.

2 Professor Titular do Dep. de Fitotecnia da Universidade Estadual do Sudoeste da Bahia, Estrada do Bem Querer, Km 4, 45100-000 Vitória da Conquista-BA. ${ }^{3}$ Professor Titular do CCTA da Universidade Estadual do Norte-Fluminense, Av. Alberto Lamego, 2000, Horto, 28015-620 Campos dos Goytacazes-RJ. ${ }^{4}$ Eng ${ }^{\mathrm{a}}$--Agr ${ }^{\mathrm{a}}$. Professora de Silvicultura do LFIT/CCTA. ${ }^{4}$ Professor de Silvicultura Tropical da Universidade Federal do Rio de Janeiro-UFRJ BR, Km 7, 23890-000 Seropédica-RJ. 


\section{INTRODUÇÃO}

A escolha da espécie a ser plantada é importante para o êxito de um programa de reflorestamento. Quando se pretende alcançar povoamentos mais produtivos, são as características das mudas a serem produzidas que indicam a qualidade das árvores adultas. Tecnologias modernas devem produzir mudas de alto padrão de qualidade, para que possam suportar as adversidades do meio, apresentar altos porcentuais de sobrevivência no campo, possibilitar a diminuição da frequiência dos tratos culturais do povoamento recém-implantado e produzir árvores com volume e qualidades desejáveis.

Alguns pesquisadores têm ressaltado a importância das raízes no desempenho das plantas após o plantio, dadas às atividades fisiológicas das quais dependem o crescimento das mudas. Wakeley (1954) reconheceu que as qualidades fisiológicas das mudas podem ser mais importantes que os efeitos de ordem morfológica. Dentre os parâmetros usados no estudo do sistema radicial, com o objetivo de avaliar a qualidade fisiológica das mudas, está o potencial de regeneração de raízes (P.R.R.). Este parâmetro foi conceituado por Stone et al. (1962) e Ritchie (1985) como a combinação do potencial de iniciação de crescimento de raízes laterais com o potencial de seu alongamento. De acordo com Parviainen (1981), o P.R.R. é uma característica que prognostica o porcentual de sobrevivência e o crescimento inicial após o plantio, visa avaliar o desempenho das mudas no campo, apresenta as vantagens de maior rapidez na obtenção dos dados e menor custo e tem maior precisão nas medições da qualidade fisiológica (Feret et al. 1985).

Quanto à interação entre P.R.R. e densidade, Brissette \& Carlson (1986), investigando o comportamento de mudas de Pinus echinata, constataram que o número de raízes novas aumentou à medida que a densidade foi menor, o que propicia maior volume radicial. Brissette \& Roberts (1984) também constataram, em mudas de Pinus taeda, que a época de retirada e as dimensões das mudas proporcionaram grande efeito no P.R.R., sendo este um valioso instrumento de avaliação dos efeitos do sítio do viveiro, das procedências de sementes, das famílias em pomares clonais, das técnicas de produção de mudas e de outros fatores que atuam no comportamento das mudas, não só no viveiro, mas também no campo, após o plantio. Brissette et al. (1988) relataram que o P.R.R., em muitas espécies, mostra estreita relação com o vigor, a tolerância à seca, os danos físicos decorrentes da retirada das mudas dos canteiros, o armazenamento e o plantio.

R. Árvore, Viçosa-MG, v.26, n.6, p.675-681, 2002
Para McTague \& Tinus (1996), o P.R.R. consistiu em um importante atributo na explicação da sobrevivência de mudas de Pinus ponderosa. Quanto aos recipientes, segundo Schmidt-Vogt (1984), deve-se priorizar a produção de mudas sem deformações radiciais, com o propósito de alcançar maiores incrementos anuais após o plantio. $\mathrm{O}$ autor ressaltou ainda que a produção de mudas em recipientes deve proporcionar a formação de fortes sistemas radiciais, com o mínimo de deformações. Também Carneiro (1987) recomendou que os recipientes não devem provocar dobras e crescimento das raízes em espiral. Alm \& Schantz-HANSEN (1974) comentaram que recipientes pequenos, como os tubetes, são inadequados, pois restringem o crescimento do sistema radicial e não proporcionam quantidade adequada de meio para o enraizamento de mudas de espécies do gênero Pinus. Segundo Mattei (1993), o tubete mostrouse inadequado para produção de mudas de Pinus taeda, induzindo à deformação das raízes laterais, podendo trazer conseqüências negativas para o crescimento futuro das mudas no campo. Parviainen (1984) recomenda o uso de blocos prensados de turfa $(60 \times 40 \mathrm{~cm})$, que possibilita o desenvolvimento do sistema radicial em sua posição natural, livre, sem confinamento ou direcionamento das raízes, usualmente provocados por paredes de recipientes. De acordo com Parviainen (1990), este sistema possibilita às mudas um favorável desenvolvimento morfológico após o plantio, apresentando um sistema radicial praticamente isento de deformações. Segundo Parviainen \& Tervo (1989), cada bloco contém 96 pontos de semeadura, espaçados de $5 \mathrm{~cm}$. Por ocasião da expedição das mudas para o plantio, os blocos são mecanicamente serrados em sentidos transversais, para individualização dos torrões que contêm as mudas. As serras promovem a poda das raízes laterais, o que possibilita o desenvolvimento radicial e o decorrente aumento do número de extremidades de raízes.

Neste sentido, o objetivo deste trabalho foi avaliar a qualidade de mudas de Pinus taeda produzidas em diferentes tipos de recipientes, com base no estudo do potencial de regeneração de raízes (P.R.R.), visando a produção de mudas com altas taxas de sobrevivência e crescimento inicial após o plantio.

\section{MATERIAL E MÉTODOS}

As sementes da espécie em estudo foram coletadas em árvores geneticamente melhoradas. A região (Três Barras-SC) onde foram conduzidos a produção das mudas 
e o experimento de campo situa-se à latitude de $26^{\circ} 07^{\prime} \mathrm{S}$, longitude $50^{\circ} 19^{\prime} \mathrm{W}$ e altitude de $775 \mathrm{~m}$, sobre o nível do mar. O clima da região, segundo a classificação de Köppen, é do tipo $\mathrm{Cfb}$, sendo a temperatura média do mês mais quente inferior a $22^{\circ} \mathrm{C}$ e a do mês mais frio superior a $10^{\circ} \mathrm{C}$, ocorrendo mais de dez geadas por ano, e a precipitação média anual é de $1.434 \mathrm{~mm}$.

Este estudo foi conduzido em três etapas: a) produção das mudas; b) avaliação do P.R.R.; e c) desempenho das mudas após o plantio.

\subsection{Produção de Mudas}

\subsubsection{Recipientes Utilizados na Produção das Mudas}

Os blocos prensados usados neste trabalho foram produzidos pela empresa finlandesa VAPO OY. e apresentavam duas espessuras, de 2 e $3 \mathrm{~cm}$, expandindo para 7 e $10 \mathrm{~cm}$, respectivamente, quando submetidos às regas normais. Cada bloco continha 96 orifícios de aproximadamente $2 \mathrm{~mm}$ de profundidade, nos quais foram colocadas as sementes, possibilitando a produção de 96 mudas, espaçadas de $5 \mathrm{~cm}$. Os blocos foram postos em caixas de material plástico rígido, com dimensões de 60 x 40 cm, com alturas iguais à espessura do material após sua expansão. Estes blocos, tão logo as mudas alcançaram o desenvolvimento ideal para o plantio, foram serrados em sentidos transversal e longitudinal, individualizando as mudas em torrões. O tipo de tubete utilizado foi o modelo cônico, com secção circular contendo quatro frisos internos longitudinais e eqüidistantes, com dimensões de $12,5 \mathrm{~cm}$ de altura, $3 \mathrm{~cm}$ de diâmetro na parte interna superior e apresentando o fundo aberto de aproximadamente $1 \mathrm{~cm}$, com $60 \mathrm{~cm}^{3}$ de capacidade volumétrica de substrato.

\subsubsection{Tratamentos}

Foram considerados cinco tratamentos, envolvendo três sistemas de produção de mudas: tratamento 1 - bloco 10 (sistema de blocos prensados com $10 \mathrm{~cm}$ de espessura, após expansão); tratamento 2 - bloco 7 (sistema de blocos prensados com $7 \mathrm{~cm}$ de espessura, após expansão); tratamento 3 - raiz nua; tratamento 4 - tubete com menor densidade (48 mudas/bandeja); e tratamento 5 - tubete com maior densidade (96 mudas/bandeja). No tratamento 4 foi feita a distribuição uniforme das mudas em cada bandeja, deixando-se uma célula correspondente à posição de um tubete entre elas, obtendo-se a densidade de
48 mudas (D48). Para o tratamento 5, distribuíram-se os tubetes em todas as células, obtendo-se a densidade de 96 mudas (D96). Cada parcela de $1 \mathrm{~m}^{2}$, correspondente ao tratamento em raiz nua, apresentou densidade média de 240 mudas.

\subsubsection{Substrato e Obtenção das Mudas}

O substrato para enchimento dos tubetes foi uma mistura de matéria orgânica humificada, à base de turfa com $\mathrm{pH}=5,9$, com os seguintes níveis de fertilidade $(\%)$ : $\mathrm{N}($ total $)=0,90 ; \mathrm{P}=2,66 ; \mathrm{K}=0,32 ;$ e matéria orgânica $=$ 36,0 . A semeadura foi feita manualmente, colocando-se três sementes em cada tubete e também no local específico de cada bloco prensado. Como cobertura morta, foi espalhada uma camada de $1 \mathrm{~cm}$ de acículas secas picadas de pinus. As regas foram efetuadas diariamente em todo o experimento. Para o tratamento referente às mudas em raiz nua foram escolhidas, de forma aleatória, seis parcelas, em seis diferentes canteiros de um viveiro comercial. O substrato utilizado correspondeu ao próprio solo do viveiro, preparado através das operações de aração, gradagem, emprego de enxada rotativa, calagem e aplicação de adubação mineral e orgânica. As mudas foram avaliadas no viveiro, aos seis meses após a semeadura.

\subsection{Avaliação do Potencial de Regeneração de Raízes}

As mudas foram submetidas a uma lavagem cuidadosa e à poda do sistema radicial secundário, a uma distância de $4 \mathrm{~cm}$ do eixo da raiz pivotante, também podada à distância de $15 \mathrm{~cm}$ do colo. A avaliação do P.R.R. foi feita através dos seguintes parâmetros: a) número total de raízes regeneradas em aquários; b) número total de raízes regeneradas maiores que $1 \mathrm{~cm}$, em aquários; c) comprimento total de raízes regeneradas em aquários; e d) comprimento de raízes regeneradas em caixas.

\subsubsection{Teste em Aquário}

Os aquários, cinco para cada tratamento, usados para avaliação do número e comprimento de raízes tinham dimensões de $28 \mathrm{~cm}$ de comprimento, $22 \mathrm{~cm}$ de largura e $25 \mathrm{~cm}$ de altura. $\mathrm{O}$ meio de crescimento das raízes foi uma solução nutritiva completa, contendo todos os elementos minerais essenciais, com pH igual a 5,6.

R. Árvore, Viçosa-M G, v.26, n.6, p.675-681, 2002 
No Quadro 1 está a composição da solução hidropônica, segundo Machlis \& Torrey (1956), ajustada para a espécie em estudo. Instalou-se, posteriormente, uma bomba de ar conectada a cinco mangueiras, uma para cada aquário, visando a manutenção da oxigenação da solução até o final do experimento. Os aquários foram totalmente envolvidos com lona plástica de cor preta, para evitar a incidência de luz. Após 21 dias de permanência nos aquários as raízes novas, regeneradas a partir do ponto de poda, atingiram as paredes dos recipientes, sendo retiradas para avaliação do P.R.R. Imediatamente após esta retirada, as raízes regeneradas foram cortadas e colocadas sobre um plástico transparente, contendo sob ele uma folha de papel milimetrado, onde, com o auxílio de uma pinça, as raízes foram estendidas e quantificadas e seus comprimentos lidos no papel milimetrado.

\subsubsection{Teste em Caixas}

As caixas usadas como recipientes para avaliação do comprimento de raízes apresentaram dimensões de $55 \mathrm{~cm}$ de comprimento, $30 \mathrm{~cm}$ de altura e $7 \mathrm{~cm}$ de largura. Após o transplantio das mudas com as raízes podadas, as caixas foram inclinadas para um lado, até formar um ângulo aproximado de $30^{\circ}$, e revestidas por um plástico de cor preta. Na parede de vidro frontal da parte inferior de inclinação foram efetuadas as leituras do comprimento de raízes, em intervalos de sete dias, finalizando aos 90 dias. Utilizou-se um total de 20 caixas, quatro para cada um dos cinco tratamentos, sendo as quatro mudas de cada repetição espaçadas em $12 \mathrm{~cm}$.

Quadro 1 - Composição da solução hidropônica e concentrações dos macronutrientes, visando o desenvolvimento do sistema radicial de mudas de Pinus taeda

Table 1 - Composition of hydroponic solution and concentrations of the nutrients, for the development of seedlings roots of Pinus taeda

\begin{tabular}{|l|c|c|}
\hline $\begin{array}{c}\text { Substância em } \\
\text { Solução } 1 \mathrm{M}\end{array}$ & $\begin{array}{c}\text { Alíquota em } \\
1000 \mathrm{ml}\end{array}$ & $\begin{array}{c}\text { Concentração na Solução dos } \\
\text { Macronutrientes (mmol) }\end{array}$ \\
\hline $\mathrm{Ca}\left(\mathrm{NO}_{3}\right)_{2}$ & 5 & Nitrogênio 15 \\
$\mathrm{KNO}_{3}$ & 5 & Fósforo 1 \\
$\mathrm{MgSO}_{4} \cdot 7 \mathrm{H}_{2} \mathrm{O}$ & 2 & Potássio 6 \\
$\mathrm{KH}_{2} \mathrm{PO}_{4}$ & 1 & Cálcio 5 \\
Micro* & 1 & Magnésio 2 \\
FeEDTA** & 1 & Enxofre 2 \\
\hline
\end{tabular}

* 2,86 $\mathrm{g} \mathrm{H}_{3} \mathrm{BO}_{3} ; 1,81 \mathrm{~g} \mathrm{MnCl}_{2} \cdot 4 \mathrm{H}_{2} \mathrm{O} ; 0,11 \mathrm{~g} \mathrm{ZnCl} 2 ; 0,05 \mathrm{~g}$ $\mathrm{CuCl}_{2} \cdot 2 \mathrm{H}_{2} \mathrm{O}$ e $0,025 \mathrm{~g} \mathrm{Na}_{2} \mathrm{MoO}_{4} \cdot 2 \mathrm{H}_{2} \mathrm{O}$.

** 5,57 g de $\mathrm{FeSO}_{4}$. $7 \mathrm{H}_{2} \mathrm{O}$ e 7,45 g de $\mathrm{Na}_{2}$ EDTA.

\subsection{Avaliação do Desempenho das Mudas no Campo}

As mudas produzidas em blocos prensados foram individualizadas, através do corte dos blocos com uma serra manual, dez dias antes do plantio, período que permitiu o início da regeneração das raízes podadas, ainda no viveiro. O espaçamento no campo foi de 3,0 x 2,5 m, sendo o plantio efetuado manualmente. A determinação da porcentagem de sobrevivência foi feita a cada 30 dias, durante cinco meses. A cada seis meses (durante dois anos) foi efetuada uma avaliação da altura da parte aérea e do diâmetro a $5 \mathrm{~cm}$ do nível do solo.

\subsection{Procedimentos Estatísticos}

Para todos os resultados obtidos, as médias foram comparadas pelo teste de Duncan, a 5\% de probabilidade. A etapa relativa à produção das mudas foi instalada, obedecendo-se a um delineamento experimental inteiramente ao acaso, com cinco tratamentos e seis repetições. Para os tratamentos 1, 2 e 5, cada parcela consistiu de 384 mudas, distribuídas em quatro bandejas. O tratamento 4 foi constituído por oito bandejas, dado à menor densidade. Para determinação do P.R.R., por meio de testes em aquários e caixas, foram selecionadas 16 mudas (quatro/repetição), totalizando-se 80 mudas, distribuídas em cinco tratamentos. Para a etapa de campo usou-se o delineamento em blocos casualizados, adotando o mesmo número de repetições do delineamento experimental utilizado na etapa de viveiro, porém com 30 mudas por parcela, perfazendo um total de 900 mudas.

\section{RESULTADOS E DISCUSSÃO}

\subsection{Avaliação do Potencial de Regeneração de Raízes em Solução Hidropônica}

As médias referentes aos parâmetros de avaliação do P.R.R. encontram-se no Quadro 2. O maior número de raízes regeneradas foi apresentado pelas mudas produzidas em raiz nua e em blocos prensados com $10 \mathrm{~cm}$, quando comparadas àquelas produzidas em tubetes com menor (D48) e maior densidade (D96), onde foram obtidos os menores valores. As mudas produzidas em tubetes apresentaram as menores médias. Na avaliação do número de raízes regeneradas em mudas de Pinus taeda, Larsen \& Boyer (1986) constataram valores mais altos de P.R.R. em mudas com maiores diâmetros e também com maior peso de matéria seca radicial. 
Quadro 2 - Valores médios do número total e do comprimento de raízes regeneradas (acima de $1 \mathrm{~cm}$ ) de mudas de seis meses de Pinus taeda com raízes laterais podadas, 21 dias após o transplantio em solução hidropônica

Table 2 - Medium values of the total number and the length of six month-old regenerated roots seedlings (above $1 \mathrm{~cm}$ ) of Pinus taeda with pruned lateral roots, 21 days after transplanting in hydroponic solution

\begin{tabular}{|l|c|c|c|}
\hline Tratamento & № Total & $\begin{array}{c}\text { № } 1 \\
(>1,0 \mathrm{~cm})\end{array}$ & $\begin{array}{c}\text { Comprimento } \\
(\mathrm{cm})\end{array}$ \\
\hline Raiz Nua & $17,49 \mathrm{a}$ & $6,24 \mathrm{ab}$ & $10,90 \mathrm{~b}$ \\
Bloco 10 & $15,91 \mathrm{a}$ & $11,58 \mathrm{a}$ & $38,97 \mathrm{a}$ \\
Bloco 7 & $12,49 \mathrm{a} \mathrm{b}$ & $9,99 \mathrm{a}$ & $41,72 \mathrm{a}$ \\
Tubete D48 & $6,33 \mathrm{~b}$ & $2,33 \mathrm{bc}$ & $5,08 \mathrm{~b} \mathrm{c}$ \\
Tubete D96 & $5,33 \mathrm{~b}$ & $0,83 \mathrm{c}$ & $1,44 \mathrm{c}$ \\
\hline
\end{tabular}

Médias seguidas de pelo menos uma mesma letra não diferem entre si, pelo teste de Duncan, a 5\% de probabilidade.

Constatou-se ainda que as mudas produzidas nos blocos prensados com 10 e $7 \mathrm{~cm}$ apresentaram o maior número de raízes regeneradas acima de $1 \mathrm{~cm}$, em relação àquelas produzidas em tubetes, e que elas corresponderam a 72,78 e 79,98\% do número total de suas raízes regeneradas, respectivamente enquanto apenas $35,68 \%$ foi verificado para as mudas produzidas em raiz nua. Médias altas de P.R.R. também foram encontradas por Brissette \& Roberts (1984), em mudas de Pinus taeda.

As maiores médias de comprimento de raízes regeneradas foram as das mudas produzidas nos blocos prensados com 10 e $7 \mathrm{~cm}$. É provável que este material seja um meio favorável para regeneração das raízes e crescimento inicial das mudas no campo, após o plantio. As mudas produzidas em tubetes apresentaram médias de comprimento de raízes regeneradas significativamente inferiores, demonstrando baixo P.R.R. Feret et al. (1985), pesquisando mudas de Pinus taeda, concluíram que o valor mínimo de $20 \mathrm{~cm}$ de alongamento de raiz para o P.R.R. pode ser necessário para que as mudas apresentem bom desempenho no campo.

\subsection{Avaliação do Potencial de Regeneração de Raízes em Caixas}

As mudas produzidas em blocos prensados apresentaram as maiores médias (Quadro 3). Os resultados deste teste foram similares aos verificados em solução hidropônica. Portanto, as mudas que apresentaram maior capacidade de regeneração de suas raízes neste teste também apresentaram maior sobrevivência e crescimento inicial no campo, demonstrando a importância do teste na avaliação do P.R.R.

Quadro 3 - Valores médios do comprimento de raízes regeneradas de mudas de seis meses de Pinus taeda com raízes laterais podadas, 90 dias após o transplantio em caixas

Table 3 - Medium lenght values of six month-old regenerated roots seedlings Pinus taeda with pruned lateral roots, 90 days after transplanting in boxes

\begin{tabular}{|l|c|}
\hline Tratamento & Comprimento $(\mathrm{cm})$ \\
\hline Bloco 10 & $66,83 \mathrm{a}$ \\
Raiz Nua & $63,53 \mathrm{a}$ \\
Bloco 7 & $52,52 \mathrm{a}$ \\
Tubete D96 & $22,85 \mathrm{~b}$ \\
Tubete D48 & $6,94 \mathrm{c}$ \\
\hline
\end{tabular}

Médias seguidas de pelo menos uma mesma letra não diferem entre si, pelo teste de Duncan, a 5\% de probabilidade.

\subsection{Desempenho das Mudas no Campo}

\subsubsection{Sobrevivência das Mudas}

Os maiores índices de sobrevivência (Quadro 4) foram os das mudas com maior P.R.R., produzidas no sistema de blocos prensados com 10 e $7 \mathrm{~cm}$. Estes resultados estão de acordo com os obtidos por Barden et al. (1986), que trabalhando com mudas de Pinus taeda constataram que o P.R.R. mostrou ser um bom indicador da sobrevivência, especialmente em condições de estresse. Carneiro (1995) também constatou, para mudas de Pinus taeda, maiores médias de sobrevivência verificadas para as mudas produzidas no sistema de blocos prensados com $7 \mathrm{~cm}$. As mudas produzidas no sistema de raiz nua alcançaram menor taxa de sobrevivência.

Quadro 4 - Valores médios de sobrevivência de mudas de Pinus taeda, cinco meses após o plantio

Table 4 - Medium values of the survival of Pinus taeda seedlings, five months after the plantation

\begin{tabular}{|l|c|}
\hline Tratamento & Sobrevivência (\%) \\
\hline Bloco10 & 98,34 \\
Bloco 7 & 97,94 \\
Tubete D96 & 96,67 \\
Tubete D48 & 95,56 \\
Raiz nua & 88,89 \\
\hline
\end{tabular}




\subsubsection{Crescimento Inicial em Altura e Diâmetro}

O comprimento total e o número de raízes novas maiores $1 \mathrm{~cm}$ (Quadro 5) apresentaram correlações significativas com o desempenho das mudas após o plantio no campo (maiores taxas de sobrevivência e crescimento inicial). Estes resultados estão de acordo com os obtidos por Barden et al. (1986), em pesquisa com esta mesma espécie. Também Johnsen et al. (1986), trabalhando com mudas de Pinus strobus, encontraram correlação significativa entre o número e o comprimento de raízes regeneradas iguais ou maiores de $0,5 \mathrm{~cm}$, com a altura no campo.

No campo, constatou-se (Quadro 6) que as mudas produzidas no sistema de blocos prensados com $10 \mathrm{~cm}$ apresentaram as maiores médias de altura e diâmetro, enquanto as produzidas em tubetes com menor densidade (D48) apresentaram as médias mais baixas. Carneiro (1995), pesquisando mudas de Pinus taeda em blocos prensados com $7 \mathrm{~cm}$, raiz nua e tubetes, observou que as maiores médias de altura, em sítios arenoso e argiloso, foram verificadas para as mudas produzidas pelo primeiro e segundo método, respectivamente.

Quadro 5 - Correlações entre o potencial de regeneração de raízes (P.R.R.) e altura e diâmetro no campo, medidos 24 meses após o plantio de mudas de Pinus taeda

Table 5 - Correlations among root growth potential of regeneration (PRR) and field height and diameter, measured after Pinus taeda seedling plantation

\begin{tabular}{|l|c|c|c|}
\hline & № & $\begin{array}{c}\mathrm{N}^{\mathrm{o}} \\
(>1 \mathrm{~cm})\end{array}$ & $\begin{array}{c}\text { Comprimento } \\
(\mathrm{cm})\end{array}$ \\
\hline Altura (cm) & 0,30 & $0,50^{*}$ & $0,60^{*}$ \\
Diâmetro (mm) & 0,41 & $0,50^{*}$ & $0,60^{*}$ \\
\hline
\end{tabular}

* Significativo a $5 \%$ de probabilidade.

Quadro 6 - Valores médios de altura da parte aérea e diâmetro de mudas de Pinus taeda, 24 meses após o plantio

Table 6 -Medium height values of the aerial part and rootcollar diameter, Pinus taeda seedlings, 24 months after plantation

\begin{tabular}{|l|c|c|}
\hline Tratamento & Altura $(\mathrm{cm})$ & Diâmetro $(\mathrm{mm})$ \\
\hline Bloco 10 & $275,50 \mathrm{a}$ & $61,09 \mathrm{a}$ \\
Bloco 7 & $251,00 \mathrm{~b}$ & $52,86 \mathrm{~b}$ \\
Raiz Nua & $242,67 \mathrm{bc}$ & $52,75 \mathrm{~b}$ \\
Tubete D96 & $236,67 \mathrm{bc}$ & $47,01 \mathrm{c}$ \\
Tubete D48 & $226,17 \mathrm{c}$ & $45,54 \mathrm{c}$ \\
\hline
\end{tabular}

Médias seguidas de pelo menos uma mesma letra não diferem entre si, pelo teste de Duncan, a 5\% de probabilidade.
Os resultados, obtidos 24 meses após o plantio, demonstraram a superioridade das mudas produzidas por meio do sistema de blocos prensados com $10 \mathrm{~cm}$, que apresentaram maior ritmo de crescimento inicial em altura e diâmetro, evidenciando, assim, a importância desse parâmetro fisilológico na previsão do desempenho das mudas após o plantio. Por terem apresentado as maiores médias de P.R.R, foram constatados ritmos de crescimento similares para as mudas produzidas em blocos prensados com $7 \mathrm{~cm}$ e em raiz nua. Este desempenho das mudas dos blocos prensados com $7 \mathrm{~cm}$ é confirmado pelos altos valores de P.R.R. constatados na fase de avaliação de laboratório. O ritmo mais lento e os baixos valores de crescimento em altura e diâmetro no campo foram verificados em mudas produzidas em tubetes, presumivelmente por terem mostrado menor P.R.R. Carneiro (1995) encontrou as maiores médias de diâmetro em mudas de Pinus taeda produzidas em blocos prensados com $7 \mathrm{~cm}$, em comparação com as mudas produzidas em raiz nua e tubetes.

\section{CONCLUSÕES}

Após a discussão dos resultados obtidos e nas condições em que foram realizados os experimentos, conclui-se que:

1. O potencial de regeneração de raízes, avaliado em aquários e caixas, demonstrou ser um parâmetro fisiológico confiável na determinação da qualidade de mudas de Pinus taeda e previsão de seu desempenho no campo.

2. As mudas de Pinus taeda de melhor padrão de qualidade, com altos valores de P.R.R. e melhor desempenho no campo, 24 meses após o plantio, foram produzidas em blocos prensados com $10 \mathrm{~cm}$.

3. As médias mais baixas, tanto para os parâmetros de laboratório quanto para os de campo avaliados, foram verificadas em mudas produzidas em tubetes, que se mostraram inadequados para produção de mudas de Pinus taeda.

\section{REFERÊNCIAS BIBLIOGRÁFICAS}

ALM, A. A.; SCHANTZ-HANSEN, R. Tubeling research plantings in Minnesota. In: NORTH AMERICAN CONTAINERIZED FOREST TREE SEEDLING SYMPOSIUM, 1974, Denver. Proceedings... Washington, D.C. Government Printing Office, 1974. p. 384-387. (Great Plains Agric. Council Publication, 68). 
BARDEN, J. C.; FERET, P. P.; KREH, R. E. Root growth potential and outplanting performance of loblolly pine seedlings raised at 2 nurseries. In: BIENAL

SILVICULTURAL RESEARCH CONFERENCE, 4., 1986, Atlanta. Proceedings... Local: Editora, 1986. p.237-244.

(Gen. Tech. Rep. SE. USDA. For. Serv., 24).

BRISSETTE, J. C.; CARLSON, W. C. Effects of nursery density on shortleaf pine. Fort Collins, USDA, For. Serv., 1986. p.36-41. (Gen. Techn. Report. RM, 151).

BRISSETTE, J. C.; BARNETT, J. P.; GRAMLING, C. L. Root growth potential of southern pine seedlings grown at the W. Ashe nursery. SOUTHERN FOREST NURSERY ASSOCIATION, 1988.: Charleston. Proceedings... New Orleans: USDA. Forest Service. Southern Forest Experiment Station, 1988. p. 173-183.

BRISSETTE, J. C.; ROBERTS, T. C. Seedling size and lifting date effects on root growth potential of loblolly pine from two Arkansas Nurseries. Tree Planters' Notes, v. 38, n. 4, p. 34-38, 1984.

CARNEIRO, J. G. A. Produção e controle de qualidade de mudas florestais. Curitiba: UFPR/ FUPEF/UENF, 1995. $451 \mathrm{p}$.

CARNEIRO, J. G. A. Influência de recipientes e de estações de semeadura sobre o comportamento do sistema radicular e dos parâmetros morfológicos de mudas de Pinus taeda e Pinus elliottii. Curitiba, UFPR, 1987. 81 p. (Setor de Ciências Agrárias, Departamento de Silvicultura e Manejo, UFPR).

FERET, P. P.; FREYMAN, R. C.; KREH, R. E. Variation in root growth potential of loblolly pine from seven nurseries. In: IUFRO - SYMPOSIUM ON NURSERY MANAGEMENT PRACTICES FOR THE SOUTHERN PINES, 1985, Birmingham. Proceedings... Birmingham, Al.: IUFRO, 1985. p. 317-328.

JOHNSEN, K. H.; FERET, P. P.; SEILER, J. R. Root growth potential as predictor of first year field performance for non-irrigated and irrigated eastern white pine seedlings. In: Proceedings... BIENAL SILVICULTURAL RESEARCH CONFERENCE, 4.:1986, Atlanta. Asheville, U.S.D.A. Forest Serv., N. C. 1986. p. 245-250. (Gen. Techn. Report. SE. U.S.D.A. For. Serv., 24).

LARSEN, H. S.; BOYER, J. M. Root growth potential of loblolly pine (Pinus taeda) seedlings from twenty southern nurseries. Alabama: Agricultural Experiment Station, Auburn University, 1986. 16 p. (Circular, 286)

MACHLIS, L.; TORREY, J. G. A Laboratory manual of plant physiology. California: W. H. Freeman and Company, 1956. $398 \mathrm{p}$.
MATTEI, V. L. Comparação entre semeadura direta e plantio de mudas produzidas em tubetes, na implantação de povoamentos de Pinus taeda L. Curitiba: UFPR, 1993. 149 p. Tese (Doutorado em Engenharia Florestal) Universidade Federal do Paraná, 1993.

McTAGUE, J. P.; TINUS, R. The effects of seedlings quality and forest site weather on field survival of ponderosa pine. Tree Planters' Notes, n. 1, v. 47, p. 16-23, 1996.

PARVIAINEN, J. V. Qualidade e avaliação da qualidade de mudas florestais. In: SEMINÁRIO DE SEMENTES E VIVEIROS FLORESTAIS, 1., 1981, Curitiba. Anais... Curitiba: UFPR/FUPEF, 1981. p. 59-90.

PARVIAINEN, J. V. Containerized forest tree seedling prodution in Finland and the other nordic countries, In: SIMPÓSIO INTERNACIONAL SOBRE MÉTODOS DE PRODUÇÃO E CONTROLE DE QUALIDADE DE SEMENTES E MUDAS FLORESTAIS, 1984, Curitiba. Anais... Curitiba: UFPR/FUPEF, 1984. p. 403-417.

PARVIAINEN, J. V. Future trends for containerized tree seedlings production: a literature review. Silva Fennica, v. 24, n. 1, p. 93-103, 1990.

PARVIAINEN, J. V.; TERVO, L. A new approach for prodution of containerized coniferous seedlings using peat sheets coupled with root pruning. Forestry Supplement, v. 62 , p. 87-94, 1989.

RITCHIE, G. A. Root growth potential: principles, procedures and predictive ability. In: EVALUATING SEEDLING QUALITY: PRINCIPLES, PROCEDURES, AND PREDICTIVE ABILITIES OF MAJOR, 1984, Corvallis. Proceedings... Corvallis, OR: For. Res., 1985. p. 93-105.

SCHMIDT-VOGT, H. Morpho-physiological quality of forest tree seedlings: the present international status of research. In: SIMPÓSIO INTERNACIONAL SOBRE MÉTODOS DE PRODUÇÃO E CONTROLE DE QUALIDADE DE SEMENTES E MUDAS FLORESTAIS, 1984, Curitiba. Anais... Curitiba: UFPR/FUPEF, 1984. p. $366-378$.

STONE, E. C.; JENKINSON, J. L.; KRUEMAH, S. L. Root regenerating potential of douglas-fir seedling lifted at different times of the year. For. Sci., v. 5, p. 228-297, 1962.

WAKELEY, P. C. Planting the southern pines. Washington: Agriculture Monograph, 1954. 233 p. (Monograph, 18).

R. Árvore, Viçosa-M G, v.26, n.6, p.675-681, 2002 